\title{
Immunomodulatory effect of Schisandra polysaccharides in cyclophosphamide-induced immunocompromised mice
}

\author{
JIAHUI YU ${ }^{1}$, LIXIN CONG ${ }^{2}$, CHUNMEI WANG $^{1}$, HE LI $^{1}$, CHENGYI ZHANG $^{1}$, XINGANG GUAN $^{3}$, \\ PENG LIU ${ }^{3}$, YU XIE ${ }^{3}$, JIANGUANG CHEN ${ }^{1}$ and JINGHUI SUN ${ }^{1}$ \\ ${ }^{1}$ Department of Pharmacology, College of Pharmacy, Beihua University, Jilin City, Jilin 132013 ; ${ }^{2}$ Second Treatment Area of \\ Senile Disease, The First Affiliated Hospital of Changchun University of Traditional Chinese Medicine, Changchun, \\ Jilin 130021; ${ }^{3}$ Research Center for Life Sciences, Beihua University, Jilin City, Jilin 132013, P.R. China
}

Received October 26, 2017; Accepted March 1, 2018

DOI: $10.3892 /$ etm.2018.6073

\begin{abstract}
As a strategy to prevent the well-known immunosuppressant effects of cyclophosphamide (Cyp), the immunomodulatory effects of the polysaccharide extract of the fruit of Schisandra chinensis (Turcz.) Baill. were investigated in the present study. The crude Schisandra polysaccharide (SCP) was obtained by water extraction and alcohol precipitation methods. The total carbohydrate, uronic acid and protein contents were determined using the phenol-sulfuric acid, m-hydroxydiphenyl and Bradford method, respectively. The monosaccharide composition of SCP was determined by high-performance liquid chromatography. ICR mice were randomly divided into control, model, low-dose SCP $(0.4 \mathrm{mg} / 10 \mathrm{~g})$, medium-dose SCP $(0.8 \mathrm{mg} / 10 \mathrm{~g})$ and high-dose SCP $(1.6 \mathrm{mg} / 10 \mathrm{~g})$ groups. The mice in the SCP groups were intragastrically administered SCP once a day for 21 days and those from the control and model groups were administered the same volume of distilled water. Subsequently, the mice in the model and SCP groups were intraperitoneally injected with Cyp $(20 \mathrm{mg} / \mathrm{kg})$ once a day for 5 days. The mouse leukocyte count in the peripheral blood as well as thymus and spleen indexes were determined, and the phagocytic function of macrophages was estimated using a carbon clearance test. The thymus and spleen were histomorphologically observed. The levels of tumor necrosis factor- $\alpha$ and interferon- $\gamma$ were measured by ELISA. Furthermore, antibody formation and spleen lymphocyte proliferation were measured by the serum hemolysin and the MTT method, respectively. The apoptotic rate of splenic lymphocytes was determined by flow cytometric analysis. The results indicated that SCP prevents Cyp-induced impairment of the cellular, humoral and non-specific immunity,
\end{abstract}

Correspondence to: Professor Jianguang Chen or Dr Jinghui Sun, Department of Pharmacology, College of Pharmacy, Beihua University, 3999 Binjiang East Road, Jilin City, Jilin 132013, P.R. China

E-mail: chenjg118@sohu.com

E-mail: sunjinghui2008@126.com

Key words: Schisandra chinensis, polysaccharide, immunomodulation, cyclophosphamide and may be an auxiliary immune enhancer for the prevention of immune hypofunction.

\section{Introduction}

Malignant tumors are a serious threat to the health of patients and a burden on their families and society. Cyclophosphamide (Cyp) is one of the most widely used chemotherapeutic drugs for the treatment of malignant tumors, but due to its severe immunosuppressive adverse effect $(1,2)$, it is necessary to co-administer drugs with immunomodulatory function and food supplements with an immune-regulating function.

In recent years, the biological activity of polysaccharides has become a hot spot in research and development of drugs. Certain polysaccharides are known to have anti-oxidant, hypolipidemic, anti-hypoglycemic and anti-tumor effects, and a large number of studies have reported on the immunomodulatory effect of plant-derived polysaccharides (3-5). Schisandra, a well-known traditional medicine in China, is the dried ripe fruit of Schisandra chinensis (Turcz.) Baill; it has been applied for thousands of years and is a representative tonic Chinese herbal medicine (6). The major active components of Schisandra are its lignans and polysaccharides, and modern pharmacological studies indicate that Schisandra polysaccharide (SCP) prevents radiation-induced immune dysfunction (7), enhances innate immune responses and disease resistance against Aeromonas hydrophila in fish (8), and exerts immunomodulatory effects through Toll-like receptor 4-mediated activation of macrophages (9). However, the effect of SCP on the immune function in an animal model induced by Cyp has been rarely reported. In order to more comprehensively understand the effect of SCP on the immune system, the effect of SCP was investigated in mice with Cyp-induced immunosuppression. The present study provides a basis for the research and development of Schisandra medicines and health foods.

\section{Materials and methods}

Experimental animals and feed preparation. Male ICR mice (age, 6 weeks; weight, 18-22 g), were provided by the Changchun Institute of Biological Products Co., Ltd. [Changchun, China; certificate no. SCXK (Ji) 2016-0008]. The animals were kept 
in a specific pathogen-free laboratory with ad libitum access to food and water. The temperature was controlled at $20-24^{\circ} \mathrm{C}$, the humidity was $\sim 50 \%$ and mice were subjected to a $12 \mathrm{~h}$ light/dark cycle. The weight of mice was recorded twice a week. The animal experiments were approved by the Institutional Animal Care and Use Committee of Beihua University (Jilin, China). All of the experimental procedures were performed in accordance with the Guide for the Care and Use of Laboratory Animals (China).

Chemicals and materials. The following drugs and reagents were used in the present study: Cyp for injection (Jiangsu Shengdi Pharmaceutical Co., Ltd., Jiangsu, China); India ink (Shanghai Ruiyong Biotechnology Co., Ltd., Shanghai, China); $10 \%$ sheep red blood cell (SRBC) suspension (Beijing Bersee Science and Technology Co., Ltd., Beijing, China); concanavalin A (Con A; Sigma-Aldrich, Merck KGaA, Darmstadt, Germany); RPMI 1640 medium (Thermo Fisher Scientific, Inc., Waltham, MA, USA); and ELISA kits for tumor necrosis factor- $\alpha$ (TNF- $\alpha)$ and interferon- $\gamma$ (IFN- $\gamma$; Shanghai Lengton Bioscience Co., Ltd., Shanghai, China). All of the reagents were of analytical grade or chromatographically pure.

SCP preparation. The dried ripe fruit of Schisandra chinensis (Turcz.) Baill was purchased from Jilin Province Jian City Schisandra Planting Base and its identity was verified by Professor Fengli Li at the Department of Pharmacognosy (College of Pharmacy, Beihua University, Jilin, China).

The dried Schisandra berries $(1.5 \mathrm{~kg})$ were ground into powder, which was sieved through a 60 -mesh sieve, then immersed in 101 distilled water and soaked overnight at room temperature. Subsequently, the Schisandra-water mixture was boiled for $3 \mathrm{~h}$ to obtain the aqueous extract phase, which was concentrated with a rotary evaporator (cat. no. R206B; Shanghai Senco Technology Co., Ltd., Shanghai, China) at $80^{\circ} \mathrm{C}$ to 21 and then centrifuged at $4,500 \mathrm{x} \mathrm{g}$ for $15 \mathrm{~min}$ at $20^{\circ} \mathrm{C}$. The precipitate was discarded and the supernatant was kept; $95 \%$ ethanol was added to the supernatant to adjust the final ethanol concentration of the supernatant to $75 \%$, and the mixture was left to precipitate overnight at room temperature. Following centrifugation at $4,500 \times \mathrm{g}$ for $15 \mathrm{~min}$ at $20^{\circ} \mathrm{C}$, the precipitate was collected, washed once with $95 \%$ ethanol and anhydrous ethanol in turn, and then freeze-dried routinely to obtain a powder-like SCP. Voucher specimens were deposited at the College of Pharmacy, Beihua University (Jilin, China; sample no. 20170520-1).

Analysis of chemical properties. The total carbohydrate content of SCP was determined with the phenol-sulfuric acid method (10), with glucose as the standard. The uronic acid content was determined with the m-hydroxydiphenyl method (11), with D-galactose as the standard. The protein content was determined using the Bradford assay (12). The monosaccharide composition was determined by high-performance liquid chromatography (HPLC) (13).

Animal grouping,modelestablishmentanddrugadministration. A total of 200 mice were divided into 4 batches, with 50 mice in each batch. The mice in each batch were randomly divided (each, $n=10$ ) into a control group, model group, low-dose SCP group (SCP-L), medium-dose SCP group (SCP-M) and high-dose SCP group (SCP-H). All of the mice were allowed to acclimatize to the laboratory environment for 3 days. Mice in the SCP-L, SCP-M and SCP-H groups were intragastrically via oral gavage administered the corresponding doses of SCP aqueous solution $(0.4,0.8$ and $1.6 \mathrm{mg} / 10 \mathrm{~g})$, and those in the control group and the model group were intragastrically given the same volume of distilled water (14) $(0.2 \mathrm{ml} / 10 \mathrm{~g})$, successively for 21 days. On day 17 after the administration, the mice in the model, SCP-L, SCP-M and SCP-H group were intraperitoneally injected with Cyp $(20 \mathrm{mg} / \mathrm{kg})$, and those in the control group were injected the same volume of normal saline.

The mice in the present study were divided into 4 batches. The first batch of mice was used for observation of phagocytosis in macrophages and were injected with India ink, which was utilized only in this batch as it would interfere the results of other tests in this study (including histomorphology, optical density of TNF- $\alpha$ and IFN- $\gamma$ ) (15). The second batch were subjected to the serum hemolysin test. Mice were immunized using an intraperitoneal injection of SRBC 5 days prior to experimentation. This treatment caused an immune response to the body, meaning these mice were only appropriate for use in the serum hemolysin test (16). The third batch of mice were used for the determination of organ indexes, TNF- $\alpha$, IFN- $\gamma$ and leukocyte count as well as histomorphology. For histomorphology, spleens were fixed using $10 \%$ formalin solution at room temperature for $24 \mathrm{~h}$ and thus could not be utilized for the preparation of the splenic lymphocyte suspension. The fourth batch was used for the assessment of splenic lymphocyte proliferation and apoptosis. Mice were sterilized by placing them in alcohol and their spleens were removed under aseptic conditions for the preparation of splenic lymphocyte suspension, so they could not be used for the other experiments.

Measurement of organ indexes and histomorphology observation. At $1 \mathrm{~h}$ after the intragastric administration, the mice were anesthetized with ether and sacrificed by $\mathrm{CO}_{2}$ inhalation; death was confirmed by observing apnea over $5 \mathrm{~min}$. The thymus and spleen of the mice were isolated, and the excess tissues and fascia were stripped and then weighed. The thymus and spleen indexes were calculated according to the following equation:

\section{Organ index $=$ organ mass/animal body mass}

Subsequently, the thymus and spleen were fixed with $10 \%$ formalin solution at room temperature for $24 \mathrm{~h}$. The pathological specimens were routinely sliced $(5 \mu \mathrm{m})$, embedded in paraffin and stained with $\mathrm{H} \& \mathrm{E}$ for $10 \mathrm{~min}$ at room temperature. Pathological changes were observed under a light microscope (magnification, x100) and images were captured.

Leukocyte count in the peripheral blood. At $1 \mathrm{~h}$ after the last intragastric administration, the mice were inhalation of anesthetized with 5\% ether and blood samples were collected by removing the eyeballs. Mice were monitored for the loss of righting reflex and were maintained at a respiratory rate of 100-200 breaths/min to ensure that anesthesia was effective. The mice were sacrificed by $\mathrm{CO}_{2}$ asphyxiation, the concentration of input $\mathrm{CO}_{2}$ was $100 \%$ and the flow rate of $\mathrm{CO}_{2}$ was set at $20 \%$ of the chamber volume/min, the apnea of mice was an indicator to determine if mice had succumbed (the mice 
sacrificed in other tests used the same method). Aliquots of blood $(20 \mu \mathrm{l})$ were added to $0.38 \mathrm{ml} 2 \%$ acetic acid and mixed. The number of white blood cells in the blood was counted with a counting plate under a microscope.

Observation of phagocytosis of macrophages in mice. At $1 \mathrm{~h}$ after the last intragastric administration, the mice were anesthetized using ether (as aforementioned) and injected with $50 \%$ diluted India ink $(0.1 \mathrm{ml} / 10 \mathrm{~g})$ through their tail veins. The time $(\mathrm{t})$ of the injection was immediately recorded, and $25 \mu \mathrm{l}$ blood was taken from the inner canthus venous plexus at $\mathrm{t}=3$ and $11 \mathrm{~min}$ after the ink injection, respectively, and immediately added to $2 \mathrm{ml} 0.1 \% \mathrm{Na}_{2} \mathrm{CO}_{3}$ solution. The optical density values of the two samples taken at 3 and $11 \mathrm{~min}$ were measured at $600 \mathrm{~nm}$ wavelength by a microplate reader to obtain the absorbance values (A3 and A11, respectively), with $\mathrm{Na}_{2} \mathrm{CO}_{3}$ solution used as the control. The carbon clearance index $(\mathrm{K})$ and the phagocytic index $(\alpha)$ were calculated according to the following equations:

$$
\mathrm{K}=(\log \mathrm{A} 3-\log \mathrm{A} 11) /\left(\mathrm{t}_{11}-\mathrm{t}_{3}\right)
$$

Phagocytic index $\alpha=$ body mass/(liver weight + spleen weight) $\mathrm{x} \mathrm{K}^{1 / 3}$

Measurement of TNF- $\alpha$ and IFN- $\gamma$. At $1 \mathrm{~h}$ after the last intragastric administration, the mice were anesthetized with ether and the blood samples were collected by removing the eyeballs. The blood was centrifuged at 3,000 x g for $10 \mathrm{~min}$ at $4^{\circ} \mathrm{C}$ to obtain the serum, and TNF- $\alpha$ and IFN- $\gamma$ levels in the serum of mice were detected according to the instructions of TNF- $\alpha$ and IFN- $\gamma$ kits using a microplate reader (Infinite M200; Tecan, Maennedorf, Switzerland).

Measurement of serum hemolysin. On day 17 after the intragastric administration, the mice were immunized by

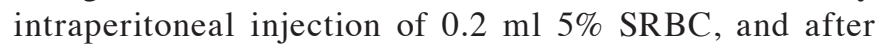
5 days, the mice were anesthetized with ether and their blood samples were collected by removing their eyeballs. The blood was centrifuged at 3,000 x g for $10 \mathrm{~min}$ to obtain the serum, which was diluted 100 times with normal saline; $0.5 \mathrm{ml}$ of the diluted serum ( $0.5 \mathrm{ml}$ normal saline in the control group) was mixed with $0.5 \mathrm{ml} 5 \%$ SRBC suspension, $0.5 \mathrm{ml} 10 \%$ complement solution (10\% guinea pig serum; Nanjing SenBeiJia Biological Technology Co., Ltd., Nanjing, China) and $0.5 \mathrm{ml}$ normal saline, another well was set as $50 \%$ hemolysis $(0.5 \mathrm{ml}$ $5 \%$ SRBC suspension with $1.5 \mathrm{ml}$ normal saline), then left to stand at $37^{\circ} \mathrm{C}$ for $30 \mathrm{~min}$, and then immediately put into an ice water bath to stop the reaction. The mixture was centrifuged at $2,000 \mathrm{x} \mathrm{g}$ for $10 \mathrm{~min}$ at $4^{\circ} \mathrm{C}$ to obtain the supernatant, and the A value of the supernatant at the wavelength of $540 \mathrm{~nm}$ was measured with a spectrophotometer. The content of serum hemolysin was reflected by the $\mathrm{HC}_{50}$ value, which was calculated according to the following equation:

$\mathrm{HC}_{50}=\mathrm{A}$ value of the sample $/ \mathrm{A}$ value of SRBC of $50 \%$ hemolysis $\mathrm{x}$ dilution factor

Splenic lymphocyte proliferation test. At $1 \mathrm{~h}$ after the last intragastric administration, mice in the different groups were sacrificed to remove their spleens under aseptic conditions. The spleen was ground from frozen and placed in $4 \mathrm{ml}$ lymphocyte separation liquid to prepare the splenic lymphocyte suspension. The suspension was centrifuged at $1,500 \mathrm{x} g$ for $10 \mathrm{~min}$ at $4^{\circ} \mathrm{C}$ and the supernatant was discarded. After the centrifugation was repeated two times, $2.5 \mathrm{ml}$ RPMI-1640 medium was added to the precipitate, $0.1 \mathrm{ml}$ of the solution was placed into a small centrifuge tube and trypan blue was added for staining. After $1 \mathrm{~min}$, half a drop of the solution was added to a counting plate, the cells were counted under a microscope and the cell density in the suspension was adjusted to $1 \times 10^{6} / \mathrm{ml}$. Of each cell suspension, $1 \mathrm{ml}$ each was added to 2 wells of a 24-well culture plate; to one well, $50 \mu \mathrm{l}$ Con A was added, and the other one was used as the control with $50 \mu$ l RPMI-1640 medium. The plates were placed in a $\mathrm{CO}_{2}$ incubator, in which the cells were cultured at $37^{\circ} \mathrm{C}$ for $72 \mathrm{~h}$. At $4 \mathrm{~h}$ prior to the end of the incubation, $0.7 \mathrm{ml}$ culture medium and $20 \mu \mathrm{l} 5 \mathrm{mg} / \mathrm{ml}$ MTT solution was added. At the end of the incubation, the supernatant was discarded and $1 \mathrm{ml}$ acidic isopropanol $(\mathrm{HCl}$ : isopropanol, 1:24) was added to each well, followed by agitation to completely dissolve the purple crystals. The optical density (OD) values were measured at the wavelength of $570 \mathrm{~nm}$ with a spectrophotometer (UV2550; Shimadzu, Kyoto, Japan). The difference in OD values between the samples that were incubated with and without Con A represented the proliferation ability of splenic lymphocytes.

Splenic lymphocyte apoptosis test. The splenic lymphocyte suspension $(1 \mathrm{ml})$ was centrifuged at $1,000 \mathrm{x}$ for $5 \mathrm{~min}$ at $4^{\circ} \mathrm{C}$, the supernatant was discarded and the precipitate was suspended in binding buffer $(0.5 \mathrm{M} \mathrm{NaCl}, 20 \mathrm{mM}$ Tris and $5 \mathrm{mM}$ Imidazole) with $200 \mu 110 \%$ Annexin V-fluorescein isothiocyanate (FITC), followed by mixing. The solution was incubated at room temperature for $10 \mathrm{~min}$, then centrifuged at $1,000 \mathrm{x} \mathrm{g}$ for $5 \mathrm{~min}$ at $4^{\circ} \mathrm{C}$ and the supernatant was discarded. The precipitate was suspended in $195 \mu 1$ Annexin V-FITC binding buffer, $10 \mu \mathrm{l}$ propidium iodide (PI) staining solution was added, the solution was gently mixed under exclusion of light and then analyzed with an Epics-XL Flow Cytometer (Beckman Coulter, Brea, CA, USA), in which Annexin V-FITC exhibited a green fluorescence and PI a red fluorescence.

Statistical methods. All values are expressed as the mean \pm standard deviation. The number of samples in each group was expressed as 'n'. SPSS software (version 19.0 for Windows; IBM Corp., Armonk, NY, USA) was used for statistical analysis. One-way analysis of variance was used for comparison between groups followed by a Dunnett's post-hoc test. $\mathrm{P}<0.05$ was considered to indicate a statistically significant difference.

\section{Results}

Chemical properties of SCP. SCP (yield, $128.2 \mathrm{~g} ; 8.55 \%$ ) was obtained from $1.5 \mathrm{~kg}$ Schisandra fruit by hot water boiling extraction followed by precipitation in $75 \%$ ethanol and conventional drying. The total carbohydrate, uronic acid, protein and monosaccharide content of SCP are listed in Table I. The results of the HPLC analysis indicated that SCP is composed of glucose $(38.0 \%)$, galactose $(36.7 \%)$, 
Table I. Chemical properties of Schisandra polysaccharide.

Monosaccharide composition (\%)

\begin{tabular}{lccccccccc}
\cline { 3 - 8 } Carbohydrate (\%) & Uronic acid (\%) & Protein $(\%)$ & Glc & Gal & GalA & Ara & Rha & Man & GlcA \\
\hline 40.60 & 24.70 & 1.51 & 38.00 & 36.70 & 12.00 & 7.30 & 4.00 & 1.20 & 0.60 \\
\hline
\end{tabular}

Glc, glucose; Gal, galactose; GalA, galacturonic acid; Ara, arabinose; Rha, rhamnose; Man, mannose; GlcA, glucuronic acid.
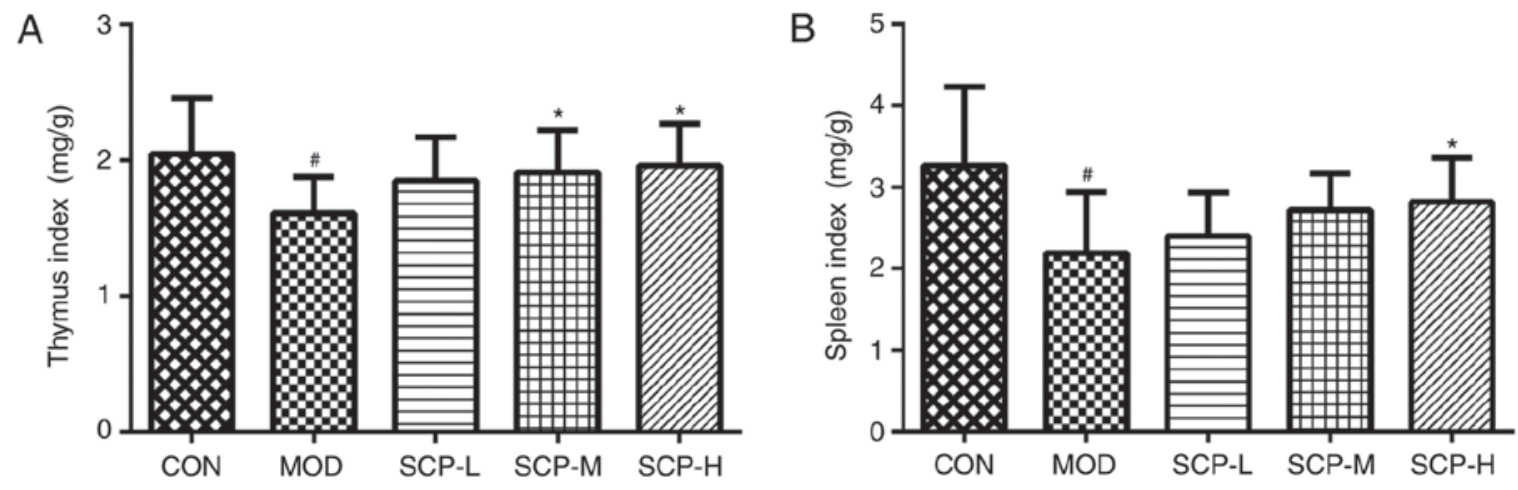

Figure 1. Effect of SCP on (A) thymus and (B) spleen indexes of the mice. Values are expressed as the mean \pm standard deviation ( $\mathrm{n}=10) .{ }^{*} \mathrm{P}<0.05$ vs. $\mathrm{CON} ;{ }^{*} \mathrm{P}<0.05$ vs. MOD. Groups: CON, control group; MOD, model group; SCP-L, low-dose SCP group (0.4 mg/10 g); SCP-M, medium-dose SCP group $(0.8 \mathrm{mg} / 10 \mathrm{~g})$; SCP-H, high-dose SCP group (1.6 mg/10 g). SCP, Schisandra polysaccharide.
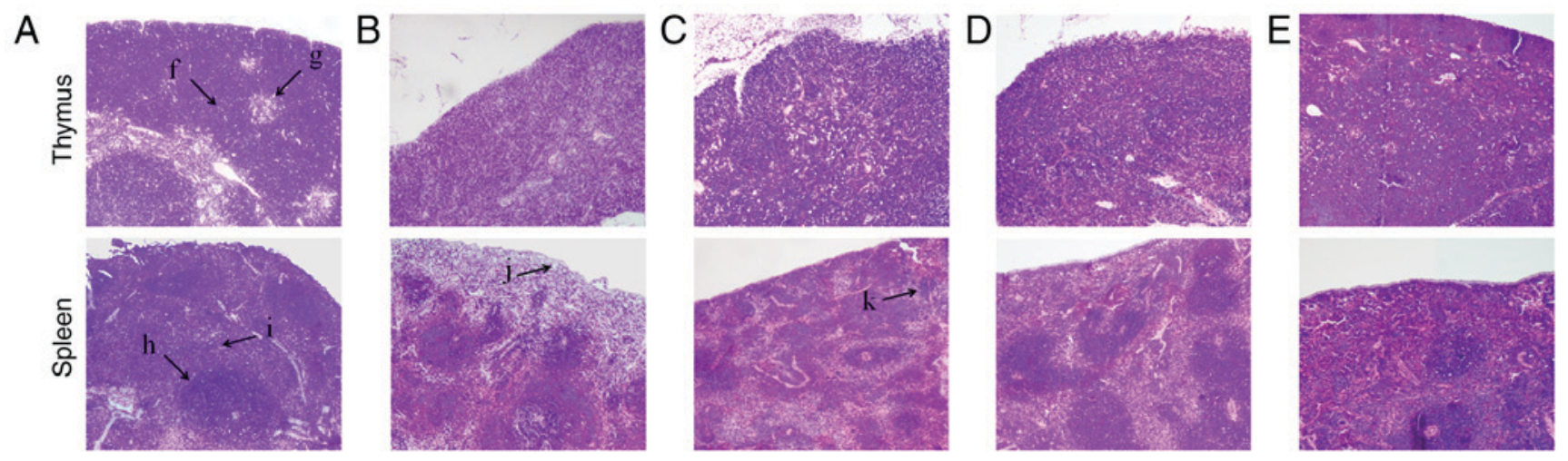

Figure 2. Effects of SCP on histomorphological changes in thymus and spleen of mice (hematoxylin and eosin staining; magnification, x100). (A) CON group; (B) MOD group; (C) SCP-L group; (D) SCP-M group; (E) SCP-H group. The arrows indicate the following features: f, cortex; g, medulla; h, white medulla; i, red medulla; j, capsule; k, splenic corpuscle. Groups: CON, control group; MOD, model group; SCP-L, low-dose SCP group (0.4 mg/10 g); SCP-M, medium-dose SCP group (0.8 mg/10 g); SCP-H, high-dose SCP group (1.6 mg/10 g); SCP, Schisandra polysaccharide.

galacturonic acid (12.0\%), arabinose (7.3\%), rhamnose (4.0\%), mannose $(1.2 \%)$ and glucuronic acid $(0.6 \%)$.

Effects of SCP on organ index and histomorphology. As indicated in Fig. 1, the thymus and spleen index of mice in the model group was significantly decreased compared with that in the control group $(\mathrm{P}<0.05)$. Furthermore, compared with that in the model group, the spleen index in the SCP-H group, and the thymus index in the SCP-M and SCP-H groups, was significantly increased $(\mathrm{P}<0.05)$.

As presented in Fig. 2, histomorphological examination (magnification, x100) indicated that the lobular beam, and the size and shape of the thymic cortex were normal, the medulla inside each cortex was visible, and the medullar structure was clear in the control group. Compared with that in the control group, the number of lymphocytes in the thymus cortex was reduced, the boundary between the medulla and cortex was not clear, and the size of the medulla was decreased in the model group. Compared with that in the model group, the boundary between the cortex and medulla was clear in the SCP-H and SCP-M groups, and the boundary between the cortex and medulla was not clear in the SCP-L group, but slightly better than that in the model group.

The spleen was also histomorphologically examined (Fig. 2). The morphology and the number of splenic corpuscles were normal, and the boundary between the red medulla and white medulla was clear in the control group. Compared with that in the control group, the number of splenic corpuscles was 
A

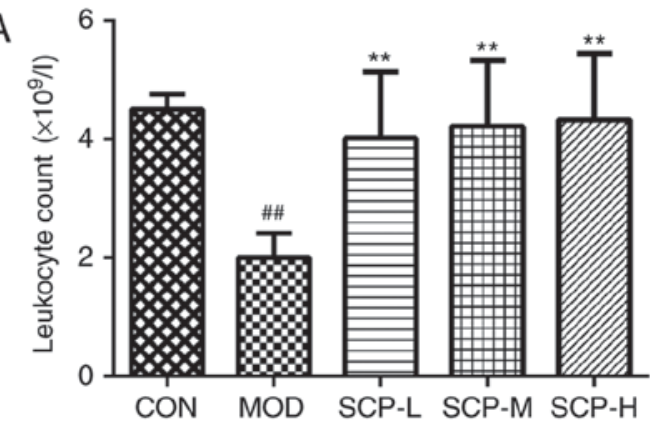

C

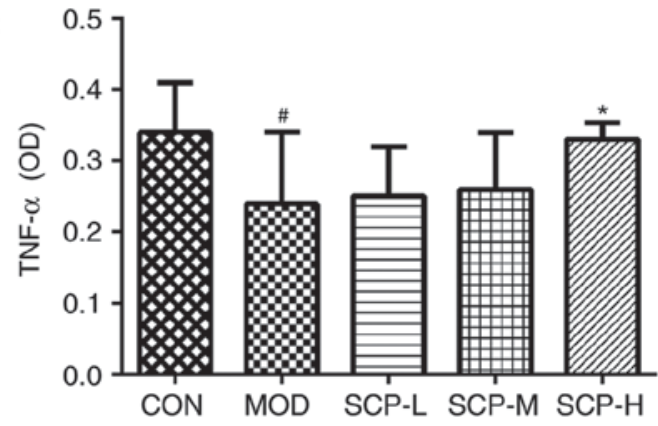

E

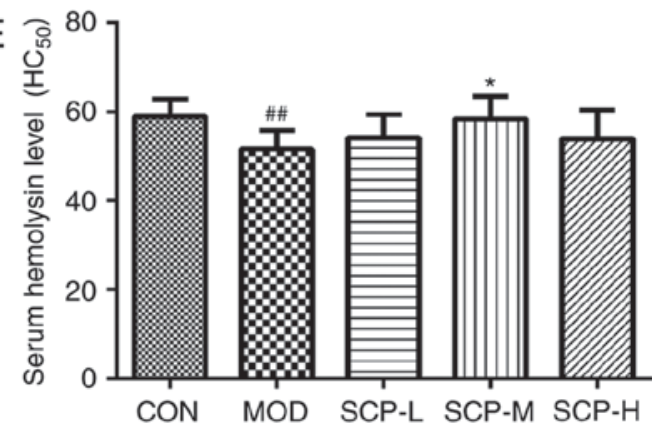

B

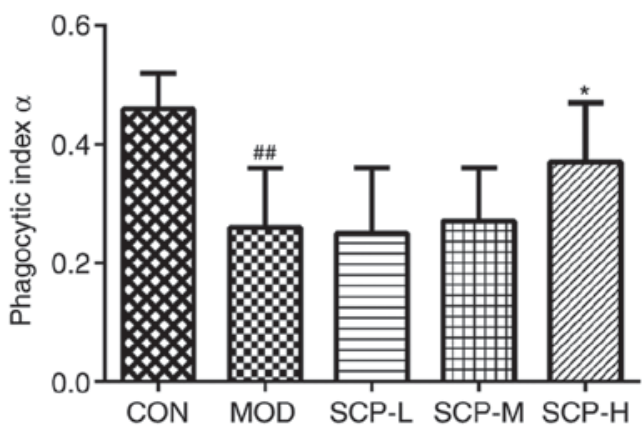

D

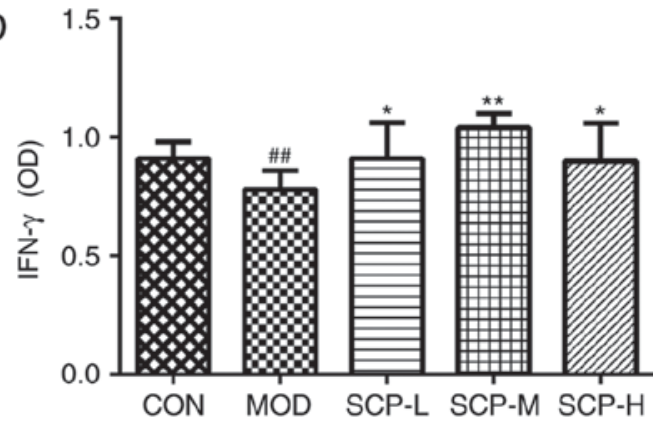

$\mathrm{F}$

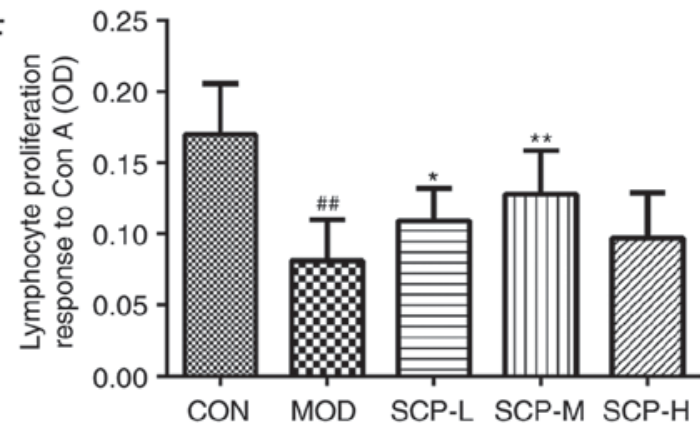

Figure 3. Effects of SCP on (A) leukocyte count in peripheral blood, (B) phagocytosis of macrophages, (C) TNF- $\alpha$, (D) IFN- $\gamma$, (E) serum hemolysin level and (F) splenic lymphocyte proliferation. Values are expressed as the mean \pm standard deviation. ${ }^{\# P}<0.05,{ }^{\# \#} \mathrm{P}<0.01$ vs. CON; ${ }^{*} \mathrm{P}<0.05$, ${ }^{* *} \mathrm{P}<0.01$ vs. MOD. Groups: CON, control group; MOD, model group; SCP-L, low-dose SCP group (0.4 mg/10 g); SCP-M, medium-dose SCP group (0.8 mg/10 g); SCP-H, high-dose SCP group (1.6 mg/10 g); SCP, Schisandra polysaccharide; TNF, tumor necrosis factor; IFN, interferon; OD, optical density; Con A, concanavalin A.

obviously reduced and their volume was obviously smaller in the spleen cortex near the capsule, and the volume of splenic corpuscles was obviously reduced in the area near the red medulla in the model group. The number of splenic corpuscles near the capsule was slightly reduced in the SCP-L group, the number in the SCP-H and SCP-M groups was close to that in the control group, and the demarcation of splenic corpuscles and red medulla was clear in the SCP-H and SCP-M groups.

Effects of SCP on leukocyte count in peripheral blood and phagocytosis of macrophages. As presented in Fig. 3A and B, compared with that in the control group, the number of leukocytes and the phagocytic index in the model group were significantly decreased $(\mathrm{P}<0.01)$. Compared with that in the model group, the number of leukocytes in the SCP-treated groups was significantly elevated $(\mathrm{P}<0.01)$, and the phagocytosis of macrophages in the SCP-H group was also significantly increased $(\mathrm{P}<0.05)$.

Effects of SCP on TNF- $\alpha$ and IFN- $\gamma$ levels. Compared with those in the control group, TNF- $\alpha$ and IFN- $\gamma$ levels in the model group were significantly decreased $(\mathrm{P}<0.05$ and $\mathrm{P}<0.01$; Fig. 3C and D, respectively). Furthermore, compared with those in the model group, the TNF- $\alpha$ levels in the SCP-H group were significantly elevated $(\mathrm{P}<0.05)$, and IFN- $\gamma$ levels in the SCP-L, SCP-M and SCP-H groups were also significantly increased $(\mathrm{P}<0.05, \mathrm{P}<0.01$ and $\mathrm{P}<0.05$, respectively).

Effects of SCP on serum hemolysin and spleen lymphocyte proliferation. As indicated in Fig. 3E, compared with that in the control group, the $\mathrm{HC}_{50}$ value in the model group was significantly decreased $(\mathrm{P}<0.01)$, indicating that Cyp significantly reduces the level of serum hemolysin to inhibit the humoral immune function in mice. Compared with those in the model group, the $\mathrm{HC}_{50}$ values in the SCP-treated groups exhibited an increasing trend, and the increase in the SCP-M group was statistically significant $(\mathrm{P}<0.05)$.

Compared with that in the control group, the proliferation of lymphocytes in the model group was significantly decreased $(\mathrm{P}<0.01)$. Compared with that in the model group, the proliferation of lymphocytes in the SCP-L and SCP-M groups was significantly increased $(\mathrm{P}<0.05$ and $\mathrm{P}<0.01$, respectively), 

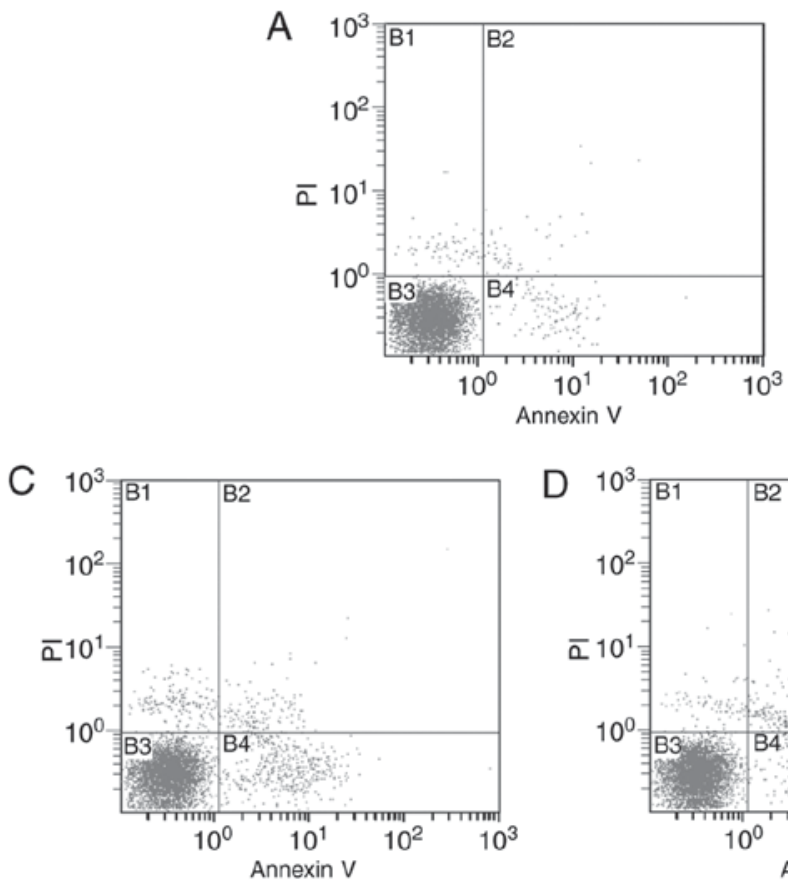
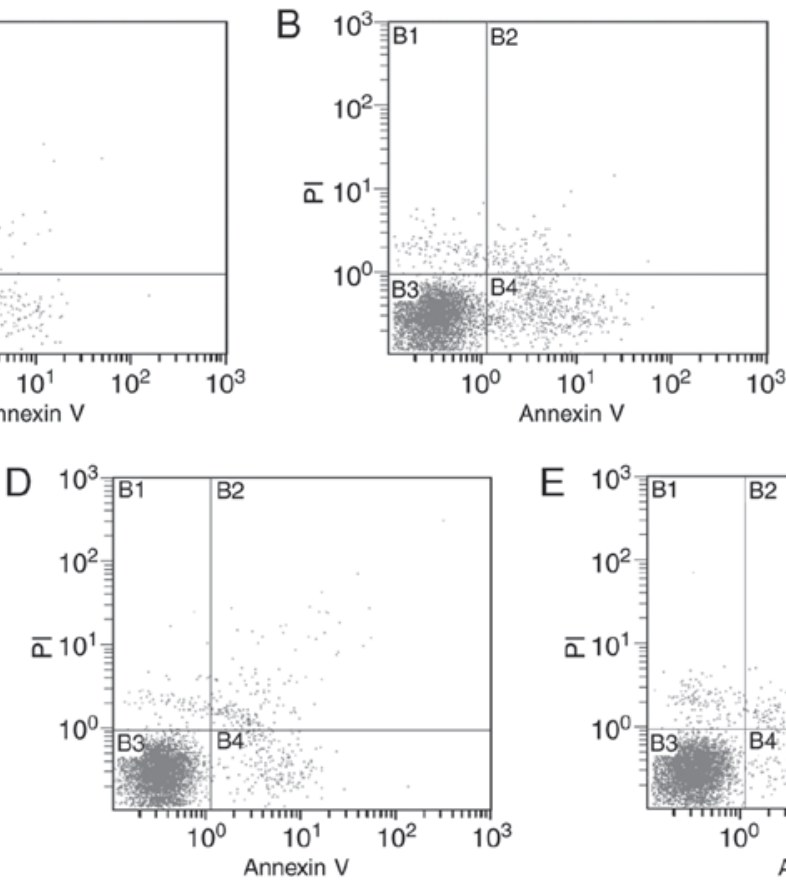

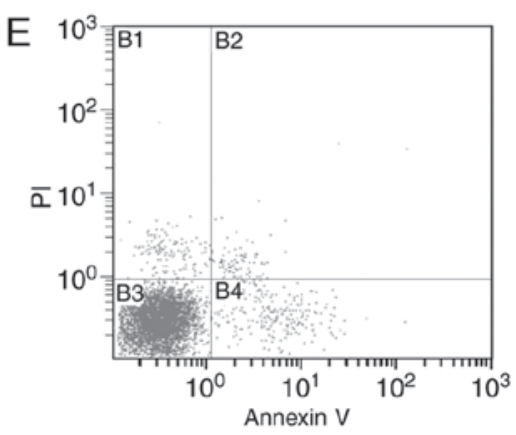

Figure 4. Effects of SCP on splenic lymphocyte apoptosis. (A) CON group; (B) MOD group; (C) SCP-L group; (D) SCP-M group; (E) SCP-H group. Quadrants: B1, necrotic cells; B2, late apoptotic cells; B3, normal cells; B4, early apoptotic cells. Groups: CON, control group; MOD, model group; SCP-L, low-dose SCP group (0.4 mg/10 g); SCP-M, medium-dose SCP group (0.8 mg/10 g); SCP-H, high-dose SCP group (1.6 mg/10 g); SCP, Schisandra polysaccharide; PI, propidium iodide.

Table II. Effects of SCP on the apoptotic rate of splenic lymphocytes.

\begin{tabular}{lccc}
\hline Group & Early $(\%)$ & Late $(\%)$ & Total $(\%)$ \\
\hline CON & $2.18 \pm 0.27$ & $0.94 \pm 0.17$ & $3.12 \pm 0.36$ \\
MOD & $11.38 \pm 2.28^{\mathrm{a}}$ & $2.56 \pm 0.74^{\mathrm{a}}$ & $13.94 \pm 1.80^{\mathrm{a}}$ \\
SCP-L & $8.22 \pm 1.06^{\mathrm{b}}$ & $2.81 \pm 0.66$ & $11.03 \pm 1.57^{\mathrm{b}}$ \\
SCP-M & $4.35 \pm 1.08^{\mathrm{b}}$ & $3.62 \pm 0.90^{\mathrm{b}}$ & $7.97 \pm 1.30^{\mathrm{b}}$ \\
SCP-H & $4.59 \pm 0.96^{\mathrm{b}}$ & $1.69 \pm 0.54^{\mathrm{b}}$ & $6.28 \pm 0.79^{\mathrm{b}}$
\end{tabular}

Values are expressed as the mean \pm standard deviation $(\mathrm{n}=10)$. ${ }^{\mathrm{a}} \mathrm{P}<0.01$ vs. $\mathrm{CON}$; ${ }^{\mathrm{b}} \mathrm{P}<0.01$ vs. MOD. Groups: $\mathrm{CON}$, control group; MOD, model group; SCP-L, low-dose SCP group $(0.4 \mathrm{mg} / 10 \mathrm{~g})$; SCP-M, medium-dose SCP group $(0.8 \mathrm{mg} / 10 \mathrm{~g})$; SCP-H, high-dose $\mathrm{SCP}$ group $(1.6 \mathrm{mg} / 10 \mathrm{~g})$; SCP, Schisandra polysaccharide.

and that in the SCP-H group was slightly but insignificantly increased (Fig. 3F).

Effect of SCP on splenic lymphocyte apoptosis. As presented in Table II and Fig. 4, compared with those in the control group, the early, late and total apoptotic rates of lymphocytes were significantly increased in the model group $(\mathrm{P}<0.01)$. In addition, compared with those in the model group, the early and late apoptotic rates of lymphocytes were significantly decreased in the SCP-H group $(\mathrm{P}<0.01)$, and the total apoptotic rate was significantly decreased in all $\mathrm{SCP}$-treated groups $(\mathrm{P}<0.01)$.

\section{Discussion}

The thymus is the central immune organ, and the site where $\mathrm{T}$ lymphocytes develop, differentiate and mature; and thymus index reflects the weight of the thymus (17). The spleen is a peripheral immune organ and the site where mature $\mathrm{T}$ and $\mathrm{B}$ lymphocytes settle and are involved in the immune response, and in the process of immune activation, immune cell differentiation and proliferation leads to an increase of its weight (18); therefore, a reduced thymus and spleen weight signifies a reduced immune cell number and declined immune function. The present results indicated that Cyp affects the differentiation and maturation of $\mathrm{T}$ lymphocytes by interfering with the cell cycle and proliferation of lymphocytes of $\mathrm{T}$ lymphocytes to cause a reduction of thymus and spleen weight, which was significantly inhibited by SCP in immunocompromised mice induced by Cyp, thereby improving the immune status that was impaired by Cyp and enhancing the immunogenic capacity of the mice.

The thymus is an important lymphoid organ in the body; its surface is covered by a connective tissue membrane, and the connective tissue stretches into the essence of the thymus to divide it into numerous incompletely separated lobules (19). The spleen is the largest lymphoid organ in the body, and its structure is similar to that of lymph nodes. The spleen is covered by a layer of connective tissue, and is divided into red medulla and white medulla. Tissue inflammation may be detected by histomorphological observation of thymus and spleen, and an abnormal morphology of these tissues may reflect lesions of immune organs, likely accompanied by a decrease of immune function. The histomorphological observation performed in the present study revealed that Cyp caused damage to thymus 
and spleen tissues due to their abnormal morphology, which was significantly inhibited by SCP.

Leukocytes are an important part of the body's defense system and counting the number of leukocytes is a valid method to evaluate immune function (20). A decrease in the number of leukocytes causes disorders of certain immune factors, leading to the decrease of immune function. Studies have reported that Cyp inhibits hematopoietic function of bone marrow to reduce the number of leukocytes (21). The results of the present study indicated that SCP significantly inhibits the decrease in leukocytes in mice immunocompromised with Cyp.

Macrophages, an important type of immune cell, have a key role in the immune response and host defense, and mediate inflammation in non-specific immunity by phagocytosis to kill and clear away pathogens and foreign bodies $(22,23)$. It is known that Cyp significantly lowers the phagocytosis of mouse macrophages (2). The present study indicated that SCP significantly preserves the function of macrophages of Cyp-induced immunocompromised mice. The study by Zhao et al (9) also indicated that Schisandra significantly enhanced the phagocytic function of macrophages in the reticuloendothelial system of mice.

Cytokines are small-molecule soluble proteins synthesized by stimulating immune cells and certain non-immune cells, of which TNF- $\alpha$, a cytokine naturally generated by the response of macrophages to bacterial infections or other immunogens, directly causes death of cancer cells (24), and IFN- $\gamma$, a multifunctional protein, is produced by monocytes and lymphocytes (25). TNF- $\alpha$ and IFN- $\gamma$ have an important role in the differentiation and regulation of immune cells, and the decrease in the content of TNF- $\alpha$ and IFN- $\gamma$ may cause disorders of immune factors, leading to a decrease of immune function. It has also been reported that Cyp significantly reduces TNF- $\alpha$ and IFN- $\gamma$ levels in mice, resulting in the inhibition of immune function (26). The present results indicating that SCP inhibited the Cyp-induced reduction the levels of TNF- $\alpha$ and IFN- $\gamma$ to improve the immune function of mice.

Serum hemolysin, a specific antibody produced by B lymphocytes in touch with red cell antigens, hemolyzes red blood cells. The level of serum hemolysin reflects the proliferation and differentiation of B cells, and their specific antibody secretion into the blood after contact with the specific antigen SRBC in the body. The determination of serum hemolysin levels of SRBC-immunized animals may be used to evaluate the function of humoral immunity (27). The present results suggested that SCP significantly increases the serum hemolysin levels to enhance the humoral immune function in Cyp-induced immunocompromised mice.

The proliferation ability of lymphocytes is an important index to evaluate the function of $\mathrm{T}$ lymphocytes in the body. $\mathrm{T}$ lymphocytes induce lymphoblast cells to proliferate after stimulation with mitogen or specific antigens, and the proliferative rate reflects the activity of T lymphocytes. When a blast cell proliferation response occurrs after $\mathrm{T}$ lymphocytes are stimulated by Con A, the proliferating cells metabolize MTT through mitochondrial hydrolytic enzymes to produce violet formazan crystals, whose OD value reflects the proliferation of lymphocytes (28). The present study indicated that Cyp significantly lowers the proliferation ability of lymphocytes cells and that SCP significantly enhances the immune function of splenic lymphocytes in immunocompromised mice induced by Cyp.

Lymphocyte apoptosis is associated with the autoimmune system itself, and the occurrence and treatment of immunity-associated diseases, so that the regulation of lymphocyte apoptosis has become a hot topic in current immunology research $(29,30)$. Therefore, splenic lymphocyte apoptosis was measured in the present study to evaluate changes in immune function. An increase in the number of lymphocyte apoptosis causes immune dysfunctions, including immunodeficiency. The present results indicated that Cyp significantly promotes apoptosis of splenic T lymphocytes in mice and that different doses of SCP had anti-apoptotic effects on these T lymphocytes induced by Cyp. Combined with the results of the splenic index and histological examination, it is suggested that the spleen atrophy and morphological changes observed may be due to apoptosis of lymphocytes induced by Cyp, and SCP may improve the immune function of the spleen by inhibiting the apoptosis of splenic lymphocytes.

In conclusion, the results of the present study suggest that SCP enhances the immune function, improves atrophy of the lymphoid organs thymus and spleen, reduces histopathological changes of thymus and spleen of immunocompromised mice, increases the number of leukocytes, promotes the formation of hemolysin and the transformation of lymphocytes, increases the phagocytic function of macrophages, elevates the levels of the immune factors TNF- $\alpha$ and IFN- $\gamma$ and inhibits apoptosis of splenic lymphocytes. The present study used a mouse model to demonstrate that the daily intake of a certain amount of SCP may be an effective way to inhibit Cyp-induced decreases in immune function, which may provide a basis for the research and development of SCP as an effective auxiliary immune enhancing agent. Furthermore, the mechanisms underlying the immunomodulatory effects of SCP should be investigated in future studies.

\section{Acknowledgements}

Not applicable.

\section{Funding}

The present study was supported by the Natural Science Foundation of Jilin Province (grant nos. 20170309006YY and 20170307016YY).

\section{Availability of data and materials}

The analyzed data sets generated during the study are available from the corresponding author on reasonable request.

\section{Authors' contributions}

JSand JC conceived and designed the experiments; JY and LC erformed the animal experiments; $\mathrm{CW}$ and HL performed the data detection; $\mathrm{CZ}$ and XG contributed to the Schisandra polysaccharides preparation; PL and YX analysed the data. The final version of the manuscript has been read and approved by all authors, and each author believes that the manuscript represents honest work.. 


\section{Ethical approval and consent to participate}

The animal experiments were approved by the Institutional Animal Care and Use Committee of Beihua University (Jilin, China).

\section{Consent for publication}

Not applicable.

\section{Competing interests}

The authors declare that they have no competing interests.

\section{References}

1. Althouse R, Huff J, Tomatis L and Wilbourn J: Chemicals and industrial processes associated with cancer in humans. IARC Monographs Volumes 1 to 20. IARC Monogr Eval Carcinog Risk Chem Hum Suppl 20: 1-71, 1979.

2. Cheng D, Wan Z, Zhang X, Li J, Li H and Wang C: Dietary Chlorella vulgaris ameliorates altered immunomodulatory functions in cyclophosphamide-induced immunosuppressive mice. Nutrients 9: pii: E708, 2017.

3. Pan G, Xie Z, Huang S, Tai Y, Cai Q, Jiang W, Sun J and Yuan Y: Immune-enhancing effects of polysaccharides extracted from Lilium lancifolium Thunb. Int Immunopharmacol 52: 119-126, 2017.

4. Bo R, Sun Y, Zhou S, Ou N, Gu P, Liu Z, Hu Y, Liu J and Wang D: Simple nanoliposomes encapsulating Lycium barbarum polysaccharides as adjuvants improve humoral and cellular immunity in mice. Int J Nanomedicine 173: 6289-6301, 2017.

5. Sheng X, Yan J, Meng Y, Kang Y, Han Z, Tai G, Zhou Y and Cheng H: Immunomodulatory effects of Hericium erinaceus derived polysaccharides are mediated by intestinal immunology. Food Funct 8: 1020-1027, 2017.

6. Panossian A and Wikman G: Pharmacology of Schisandra chinensis Bail: An overview of Russian research and uses in medicine. J Ethnopharmacol 118: 183-212, 2008.

7. Zhao LM, Jia YL, Ma M, Duan YQ and Liu LH: Prevention effects of Schisandra polysaccharide on radiation-induced immune system dysfunction. Int J Biol Macromol 76: 63-69, 2015.

8. Wang E, Chen X, Wang K, Wang J, Chen D, Geng Y, Lai W and Wei X: Plant polysaccharides used as immunostimulants enhance innate immune response and disease resistance against Aeromonas hydrophila infection in fish. Fish Shellfish Immunol 59: 196-202, 2016.

9. Zhao T, Feng Y, Li J, Mao R, Zou Y, Feng W, Zheng D, Wang W, Chen Y, Yang L and Wu X: Schisandra polysaccharide evokes immunomodulatory activity through TLR 4-mediated activation of macrophages. Int J Biol Macromol 65: 33-40, 2014.

10. Nowotny A: Carbohydrate determination by phenol-sulfuric acid. In: Basic Exercises in Immunochemistry. Springer, Berlin, pp171-173, 1979 .

11. Murado MA, Vázquez JA, Montemayor MI, Cabo ML and del Pilar González M: Two mathematical models for the correction of carbohydrate and protein interference in the determination of uronic acids by the m-hydroxydiphenyl method. Biotechnol Appl Biochem 41: 209-216, 2005.

12. Bradford MM: A rapid and sensitive method for the quantitation of microgram quantities of protein utilizing the principle of protein-dye binding. Anal Biochem 72: 248-254, 1976.

13. Zhang X, Yu L, Bi H, Li X, Ni W, Han H, Li N, Wang B, Zhou Y and Tai G: Total fractionation and characterization of the water-soluble polysaccharides isolated from Panax ginseng C. A. Meyer. Carbohyd Polym 77: 544-552, 2009.
14. Xu SY, Bian RL and Chen X: Methodology of pharmacological experiment. 3rd edition. Beijing People's Medical Publishing House, p179, 2002.

15. Jayathirtha MG and Mishra SH: Preliminary immunomodulatory activities of methanol extracts of Eclipta alba and Centella asiatica. Phytomedicine 11: 361-365, 2004.

16. Duan BW, Li Y, Liu X and Yang YJ: Effect of polysaccharides in processed Sibiraea on immunologic function of immunosuppression mice. Zhongguo Zhong Yao Za Zhi 35: 1466-1469, 2010 (In Chinese).

17. Zdrojewicz Z, Pachura E and Pachura P: The thymus: A forgotten, but very important organ. Adv Clin Exp Med 25: 369-375, 2016.

18. Kraus MD: Splenic histology and histopathology: An update. Semin Diagn Pathol 20: 84-93, 2003.

19. Flomerfelt FA, El Kassar N, Gurunathan C, Chua KS, League SC Schmitz S, Gershon TR, Kapoor V, Yan XY, Schwartz RH and Gress RE: Tbata modulates thymic stromal cell proliferation and thymus function. J Exp Med 207: 2521-2532, 2010.

20. Lämmermann T and Germain RN: The multiple faces of leukocyte interstitial migration. Semin Immunopathol 36: 227-251, 2014.

21. Zhang PP, Meng ZT, Wang LC, Guo LM and Li K: Astragalus polysaccharide promotes the release of mature granulocytes through the L-selectin signaling pathway. Chin Med 10: 17, 2015.

22. Wynn TA and Vannella KM: Macrophages in tissue repair, regeneration, and fibrosis. Immunity 44: 450-462, 2016.

23. Yu Q, Nie SP, Wang JQ, Yin PF, Huang DF, Li WJ and Xie MY: Toll-like receptor 4-mediated ROS signaling pathway involved in Ganoderma atrum polysaccharide-induced tumor necrosis factor- $\alpha$ secretion during macrophage activation. Food Chem Toxicol 66: 14-22, 2014.

24. Kouakou K, Schepetkin IA, Jun S, Kirpotinal LN, Yapi A, Khramova DS, Pascual DW, Ovodov YS, Jutila MA and Quinn MT: Immunomodulatory activity of polysaccharides isolated from Clerodendrum splendens: Beneficial effects in experimental autoimmune encephalomyelitis. BMC Complement Altern Med 13: 149, 2013.

25. Yang LC, Lu TJ, Hsieh CC and Lin WC: Characterization and immunomodulatory activity of polysaccharides derived from Dendrobium tosaense. Carbohydr Polym 111: 856-863, 2014.

26. Huang C, Song K, Ma W, Ding J, Chen Z and Zhang M: Immunomodulatory mechanism of Bushen Huoxue Recipe alleviates cyclophosphamide-induced diminished ovarian reserve in mouse model. J Ethnopharmacol 208: 44-56, 2017.

27. Sheng X, Yan J, Meng Y, Kang Y, Han Z, Tai G, Zhou Y and Cheng H: Immunomodulatory effects of Hericium erinaceus derived polysaccharides are mediated by intestinal immunology. Food Funct 8: 1020-1027, 2017.

28. Xing J, Xiao Y, Tang X, Sheng X and Zhan W: Inhibition of Cyclosporine A or rapamycin on $\mathrm{T}$ lymphocyte counts and the influence on the immune responses of B lymphocytes in flounder (Paralichthys olivaceus). Fish Shellfish Immunol 66: 78-85, 2017.

29. Liu C, Sun Z, Xu Z, Liu T, Pan T and Li S: Down-regulation of microRNA-155 promotes selenium deficiency-induced apoptosis by tumor necrosis factor receptor superfamily member $1 \mathrm{~B}$ in the broiler spleen. Oncotarget 8: 58513-58525, 2017.

30. Pinhu L, Qin Y, Xiong B, You Y, Li J and Sooranna SR: Overexpression of Fas and FasL is associated with infectious complications and severity of experimental severe acute pancreatitis by promoting apoptosis of lymphocytes. Inflammation 37 : 1202-1212, 2014

This work is licensed under a Creative Commons Attribution-NonCommercial-NoDerivatives 4.0 International (CC BY-NC-ND 4.0) License. 